\title{
Factores de riesgo asociados al ingreso a unidad de cuidados intensivos en pacientes pediátricos hospitalizados por dengue en Cali, Colombia
}

Risk factors associated with Intensive Care Unit admission in pediatric patients hospitalized for dengue in Cali, Colombia

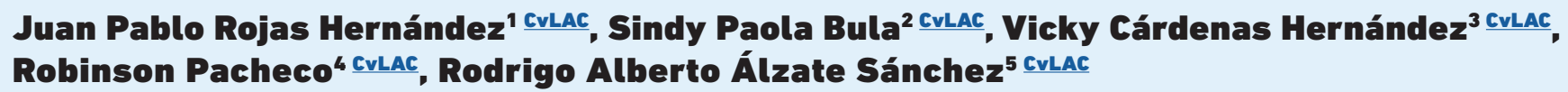

Fecha correspondencia: Recibido: septiembre 15 de 2019. Revisado: diciembre 11 de 2019. Aceptado: abril 27 de 2020.

Forma de citar:

Rojas Hernández JP, Bula SP, Cárdenas Hernández V, Pacheco R, Álzate Sánchez RA. Factores de riesgo asociados al ingreso a unidad de cuidados intensivos en pacientes pediátricos hospitalizados por dengue en Cali, Colombia. Rev CES Med 2020; 34(2): 93-102.

Open access

(c) Derecho de autor

Licencia creative commons

Ética de publicaciones

Revisión por pares

Gestión por Open Journal System DOl: http://dx.doi.org/10.21615/ cesmedicina.34.2.1

ISSN 0120-8705

e-ISSN 2215-9177

Comparte

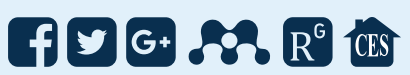

\section{Resumen}

Introducción: el dengue es la arbovirosis de propagación más rápida en todo el mundo; anualmente 390 millones de personas son infectadas. Objetivo: identificar las manifestaciones clínicas y características demográficas asociadas a ingreso a unidad de cuidados intensivos en una institución pediátrica de Cali, Colombia, entre enero 2015 y diciembre 2016. Materiales y métodos: estudio de casos y controles anidado en una cohorte. Caso: paciente mayor de 28 días y menor de 18 años, hospitalizado en la unidad de cuidados intensivos pediátricos con diagnóstico confirmado de dengue con signos de alarma o dengue grave, sumado a serología positiva para IgM y/o NS1. Control: paciente de igual edad y diagnóstico confirmado de dengue, sin ingreso a la unidad de cuidas intensivos. Resultados: se evaluaron 24 casos y 176 controles, la mediana de edad en meses fue 142 vs.106 (RIC: 59-142). El $83 \%$ procedía del área de urbana de Cali y $53 \%$ era de sexo masculino. Presentar derrame pleural OR 3,4 (IC 95\% 1,2-9,8) y alteraciones cardiovasculares OR 4,7 (IC 95\% 1,7-13,1), incrementaron la probabilidad de ingreso a unidad de cuidados intensivos pediátricos. Tres pacientes fallecieron, todos pertenecían al grupo de casos. Conclusiones: el dengue en población pediátrica tiene un comportamiento clínico inespecífico; sin embargo, identificar derrame pleural o alteraciones cardiovasculares pueden explicar un ingreso a unidad de cuidados intensivos pediátricos y alertar sobre un posible desenlace fatal.

Palabras clave: Dengue; Unidad de cuidados intensivos; Niño hospitalizado.

\section{Abstract}

Introduction: Dengue is the fastest spreading arbovirosis worldwide, 390 million people are infected annually. Objective: To identify the clinical manifestations and demographic characteristics associated with severe dengue in a pediatric institution in Cali, Colombia, between January 2015 and December 2016. Methods: Case-control study nested in a cohort. Case: patient over 28 days and under 18 years of age, hospitalized in the Pediatric Intensive Care Unit (PICU) with a confirmed diagnosis of dengue with warning signs or severe dengue, plus positive serology for IgM and / or NS1. Control: 


\section{Sobre los autores:}

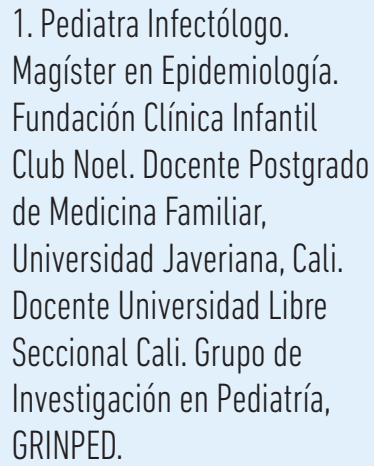

2. Médica. Hospital Tobías Puerta, Uramita.

3. Médica, Hospital Pediátrico de la Misericordia, Bogotá.

4. Maestría en Epidemiología. Grupo de Investigación en Pediatría, GRINPED. Universidad Libre Seccional Cali.

\section{Programa postdoctoral} Takemi Harvard. GrIEpiSGrupo de Investigación en Pediatría, GRINPED. Universidad Libre Seccional Cali.

La mortalidad por dengue es evitable en el $98 \%$ de los casos, estando estrechamente relacionada con la calidad en la atención del paciente. patient of equal age and confirmed diagnosis of dengue, without admission PICU. Results: 24 cases and 176 controls were evaluated, the median age in months was 142 vs. 106 (95\% Cl). Of the total, $83 \%$ proceed from the urban area of Cali. $53 \%$ of the patients were male. The multivariate analysis showed that presenting pleural effusion OR 3.4 (95\% Cl 1.2-9.8) or cardiovascular disorders OR 4,7 (95\% Cl 1.7-13,1), increased the probability of admission to PICU. Three patients died, all belonged to the case group. Conclusions Dengue in the pediatric population has a nonspecific clinical behavior, however, identifying pleural effusion and cardiovascular alterations can explain an admission to PICU and warn of a possible fatal outcome.

Keywords: Dengue; Pediatric Intensive Care Unit; Hospitalized; Children.

\section{Introducción}

El dengue es una enfermedad viral febril aguda producida por el virus del dengue y transmitida por especies de Aedes aegypti y Aedes albopictus (1). Anualmente, aproximadamente 390 millones de personas en más de 100 países están infectadas con el virus del dengue (DENV); El 70 \% de los casos ocurre en países de Asia (2).

Se reconocen cinco serotipos a nivel mundial (3), y cuatro de ellos (DENV-1 a DENV-4) son circulantes en Colombia, siendo el DENV-2 (44\%) y el DENV-1 (41\%) los más frecuentes (4).

Este estudio se realizó en Cali, Colombia, una ciudad con una población de 2470 747 personas (98,5 \% en la zona urbana), localizada a 1070 metros sobre el nivel del mar, con una temperatura media de $24,7^{\circ} \mathrm{C}$ y una precipitación anual de 1019 $\mathrm{mm}$ (5). Condiciones que explican la persistencia de mosquitos de Aedes aegypti y $A$. albopictus (6).

El espectro de manifestaciones de la enfermedad van desde procesos asintomáticos hasta cuadros severos. Es así como se definen diversas formas clínicas: dengue sin signos de alarma, dengue con signos de alarma y el dengue grave, donde se encuentran incluidos el síndrome de choque por dengue y otras complicaciones como miocarditis, encefalitis y hepatitis, asociadas con mayor mortalidad (7)(8).

En Colombia, la distribución del Aedes aegypti es generalizada y el dengue es endémico: aproximadamente 25 millones de personas en Colombia están en riesgo de contraer la enfermedad del dengue (9).

El comportamiento del dengue en Cali, Colombia ha sido endemo-epidémico con ciclos cada 3 - 5 años, que se fueron acortando hasta tener epidemias en los años 2015 y 2016. Es importante tener en cuenta que desde el 2014 está circulando en la ciudad, el virus Chikungunya y desde el 2015 el virus Zika; ambos emergentes y con sintomatología muy similar, lo cual puede causar sobre registros, especialmente en las primeras fases epidémicas del chickungunya y zika (10).

La mortalidad por dengue es evitable en el $98 \%$ de los casos, estando estrechamente relacionada con la calidad en la atención del paciente. La identificación precoz de los casos, un tratamiento eficaz y el ingreso a unidad de cuidados intensivos pediátricos (UCIP), buscan principalmente evitar la mortalidad (11)(12). 
Estudio de casos y controles anidado a una cohorte, donde participaron 200 pacientes hospitalizados por dengue.
El objetivo de este estudio fue identificar las manifestaciones clínicas y características demográficas asociadas a ingreso a unidad de cuidados intensivos pediátrico en una institución pediátrica de Cali, Colombia, entre enero 2015 y diciembre 2016.

\section{Metodología}

Estudio de casos y controles anidado a una cohorte, donde participaron 200 pacientes hospitalizados por dengue. Durante el periodo de estudio se aplicaron criterios para la clasificación en caso o control (flujograma 1).

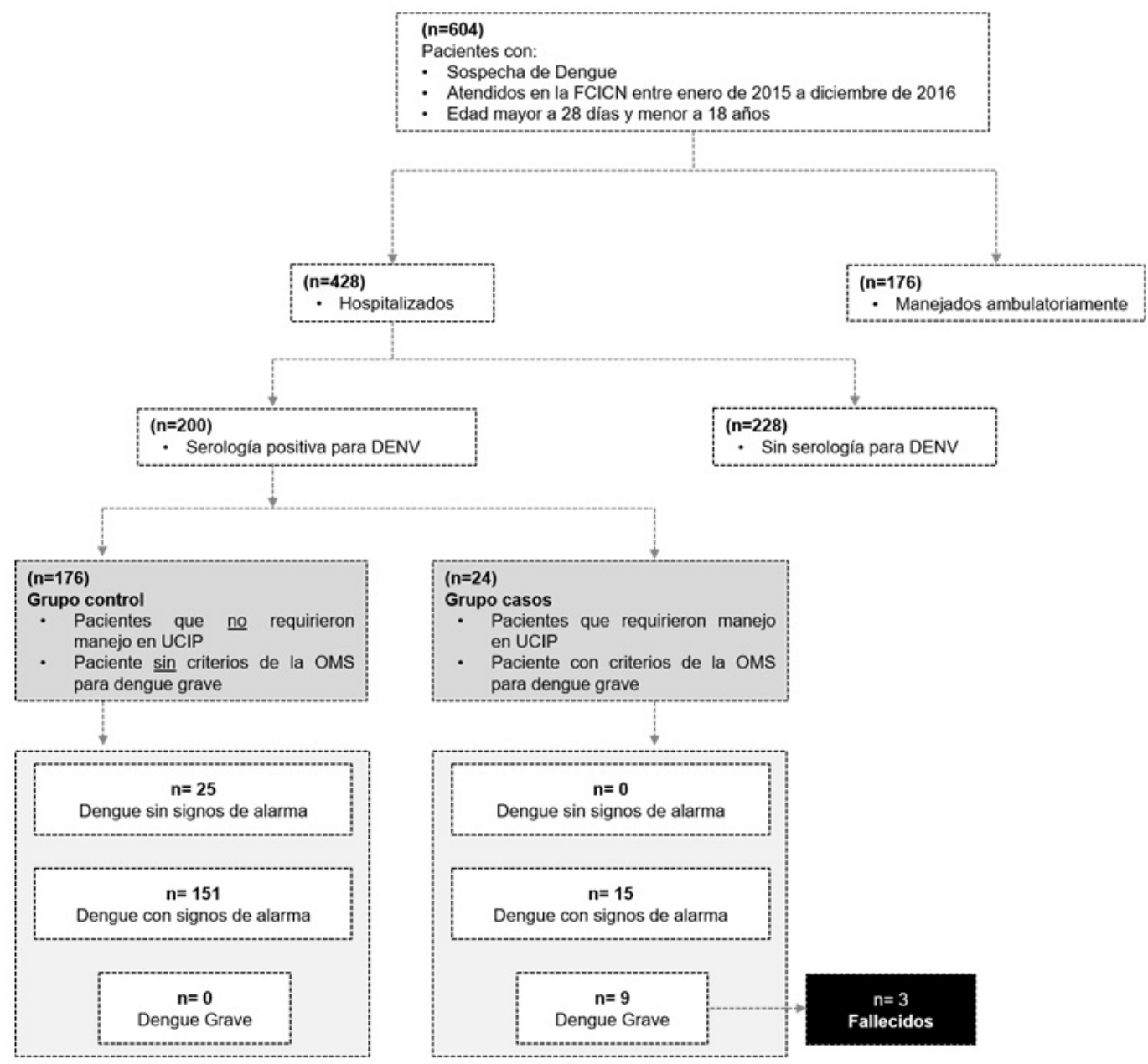

Flujograma 1. Flujo de la información sobre metodología. DENV: virus del Dengue, Serología: NS1 - IgM DENV

Caso: paciente mayor de 28 días y menor de 18 años, hospitalizado en la UCIP con diagnóstico confirmado de dengue con signos de alarma o dengue grave (según criterios de clasificación de la OMS) (12), sumado a serología positiva para IgM y/o NS1.

Control: paciente mayor de 28 días y menor de 18 años con diagnóstico confirmado de dengue sin signos de alarma, dengue con signos de alarma y dengue grave (según criterios de clasificación de la OMS), sumado a serología positiva para IgM y/o NS1, sin ingreso a unidad de cuidados intensivos pediátrico. 
Del total de pacientes $(n=200)$, el $83 \%$ procedía del área urbana de Cali y $53 \%$ eran de sexo masculino. La mediana de días de enfermedad antes de la consulta fue de tres y 98,5\% tenían fiebre al momento de la consulta.
La información fue almacenada en una base de datos electrónica (Microsoft Excel 2010, Microsoft Corp $\left.{ }^{\circledR}\right)$ mediante doble digitación comparativa. Todos los análisis estadísticos se realizaron en software Stata ${ }^{\circledR}$ (Stata Corp,2011, Stata 12 Base Reference Manual, College Station, TX, USA).

En el análisis univariado se realizó la descripción por medio de la estimación de los promedios y desviaciones estándar para las variables cuantitativas y la estimación de frecuencias y proporciones para las variables cualitativas, comprobando la normalidad con las pruebas de Kolomogorov-Smirnov o de Shapiro-Wilk si las muestras eran pequeñas $(<30)$.

Se realizó la comparación de las variables entre ambos grupos por medio de las pruebas de Chi-Cuadrado de homogeneidad $\left(\mathrm{x}^{2}\right)$ para las variables cualitativas y de t-student para las variables cuantitativas. Estableciendo como variable de exposición las características evaluadas y como variable resultado la presencia o no de dengue grave, se estimaron los "odds ratios" (OR) crudos con sus respectivos intervalos de confianza (IC) del $95 \%$.

Para ajustar por posibles factores confusores y determinar el peso que cada variable tenía se realizó una regresión logística binaria. En la construcción de los modelos se incluyeron las variables que en el análisis bivariado presentaron una significancia estadística $\leq 0,20$. El modelamiento se realizó a través de la estrategia Backward y se presentó el modelo más parsimonioso con la prueba de Hosmer-Lemeshow. Para el grado de acierto del modelo se usó la curva de ROC.

El estudio fue avalado por el Comité de Ética de la Universidad Libre seccional Cali, Colombia, según acta extraordinaria 02-2 del 27 de abril de 2015, y el acceso a los registros médicos fue autorizado por el comité de investigación de la Fundación Clínica Infantil Club Noel de Cali, Colombia.

El personal del estudio fue capacitado en buenas prácticas clínicas para la recolección y gestión de datos. El uso de registros médicos se realizó de acuerdo con la legislación sobre documentos privados en Colombia y las recomendaciones de las autoridades reguladoras nacionales e internacionales.

\section{Resultados}

Del total de pacientes ( $n=200$ ), el $83 \%$ procedía del área urbana de Cali y $53 \%$ eran de sexo masculino. La mediana de días de enfermedad antes de la consulta fue de tres (RIC: 2-4), el 98,5\% tenían fiebre al momento de la consulta. Por otra parte, $62,5 \%$ de las infecciones se clasificaron como primarias y $37,5 \%$ como secundarias (cuadros 1 y 2 ). 
Se encontró que presentar derrame pleural, alteraciones cardiovasculares, dolor abdominal y hepatomegalia, incrementaron la probabilidad de ingreso a UCIP.
Cuadro 1. Características clínicas y demográficas de la población de estudio (\%)

\begin{tabular}{|c|c|}
\hline Característica & Medida de resumen (\%) \\
\hline Edad (meses) & Mediana: 112 (RIC: 59-142) \\
\hline Sexo femenino & 53 \\
\hline Procedencia Cali & 83 \\
\hline Fiebre al momento de la consulta & 98,5 \\
\hline Dolor osteo/muscular & 51,5 \\
\hline Vómito & 58 \\
\hline Diarrea & 32,5 \\
\hline Dolor retro ocular & 12 \\
\hline Derrame pleural & 20,5 \\
\hline Alteraciones cardiovasculares & 4,5 \\
\hline Dolor abdominal & 51 \\
\hline Hepatomegalia & 29,5 \\
\hline Esplenomegalia & 2,5 \\
\hline Ascitis & 8 \\
\hline Edema & 18,5 \\
\hline Exantema & 43,5 \\
\hline Prueba de torniquete positiva $(n=33)$ & 45,5 \\
\hline NS1 positiva $(n=73)$ & 69,9 \\
\hline IgG positiva $(n=136)$ & 77,2 \\
\hline IgM positiva $(n=167)$ & 88 \\
\hline Fallecidos & 1,5 \\
\hline
\end{tabular}

RIC: Rango intercuartílico, UCIP: unidad de cuidados intensivos pediátrica

Cuadro 2. Clasificación de las infecciones primarias y secundarias

\begin{tabular}{|c|c|c|c|c|c|c|c|}
\hline Característica & Descripción & $n$ & Caso $n=24$ & Control $n=176$ & $O R$ & IC $95 \%$ & Valor $p$ \\
\hline \multirow{2}{*}{$\lg G$} & Positiva & 105 & 17 & 88 & 2,8 & $0,6-12,8$ & \\
\hline & Negativa & 31 & 2 & 29 & & & 0,879 \\
\hline \multirow{2}{*}{$\lg M$} & Positiva & 147 & 19 & 128 & \multirow{2}{*}{0,8} & \multirow{2}{*}{$0,2-3,1$} & \multirow{2}{*}{0,383} \\
\hline & Negativa & 20 & 3 & 17 & & & \\
\hline \multirow{2}{*}{ NS1 } & Positiva & 51 & 8 & 43 & \multirow{2}{*}{0,9} & \multirow{2}{*}{$0,2-3,1$} & \multirow{2}{*}{0,392} \\
\hline & Negativa & 22 & 4 & 18 & & & \\
\hline $\begin{array}{l}\text { Infecciones } \\
\text { primarias totales }\end{array}$ & $\begin{array}{l}\lg M \text { y/o NS1 } \\
\text { positivo }\end{array}$ & & \multicolumn{2}{|c|}{$125(62,5 \%)$} & & & \\
\hline $\begin{array}{l}\text { Infecciones } \\
\text { secundarias totales }\end{array}$ & $\lg G$ positivo & & \multicolumn{2}{|c|}{$75(37,5 \%)$} & & & \\
\hline
\end{tabular}

Se encontró que presentar derrame pleural, alteraciones cardiovasculares, dolor abdominal y hepatomegalia, incrementaron la probabilidad de ingreso a UCIP (cuadro 3). Las alteraciones cardiovasculares corresponden a alteraciones que abarcaron: prolongación del intervalo PR, bradicardia o derrame pericárdico. 
En el análisis multivariado se evidenció que presentar derrame pleural y alteraciones cardiovasculares OR 4,7 (IC 95\% 1,7-13,1), incrementaron la probabilidad de ingreso a unidad pediátrica de cuidados intensivos (cuadro 3 ).

Tres pacientes fallecieron, todos eran de Cali, dos tenían IgG-IgM-NS1 positivos, todos tenían previamente vómito al momento de la consulta, presentaron alteraciones cardiovasculares, dos con dolor abdominal y uno con derrame pleural.

Cuadro 3. Medidas de asociación entre variables de exposición y desenlace

\begin{tabular}{ccccccc}
\hline Característica & Descripción & $\boldsymbol{n}$ & $\begin{array}{c}\text { Caso } \\
\mathbf{n = 2 4}\end{array}$ & $\begin{array}{c}\text { Control } \\
\boldsymbol{n = 1 7 6}\end{array}$ & $\begin{array}{c}\text { OR Crudo } \\
\text { (IC 95 \%) }\end{array}$ & $\begin{array}{c}\text { OR ajustado } \\
\text { (IC 95 \%) }\end{array}$ \\
\hline Sexo & Hombre & 106 & 9 & 97 & $0,4(0,2-1,3)$ & $0,4(0,2-1,1)$ \\
\cline { 2 - 6 } & Mujer & 94 & 15 & 79 & & \\
\hline Dolor osteo/muscular & Presente & 103 & 16 & 87 & $2,0(0,8-5,8)$ & $2,3(0,8-6,3)$ \\
\hline Diarrea & Presente & 65 & 11 & 54 & $1,9(0,7-4,9)$ & $1,9(0,7-5,4)$ \\
\hline $\begin{array}{c}\text { Derrame pleural } \\
\text { Alteraciones }\end{array}$ & Presente & 41 & 13 & 28 & $6,2(2,3-17)$ & $3,4(1,2-9,8)$ \\
\hline $\begin{array}{c}\text { cardiovasculares } \\
\text { Dolor abdominal }\end{array}$ & Presente & 47 & 14 & 33 & $6,1(2,3-16,5)$ & $4,7(1.7-13,1)$ \\
\hline
\end{tabular}

\section{Discusión}

Se requieren realizar extensos estudios serológicos de la seroprevalencia de anticuerpos contra el virus del dengue para proporcionar una estimación más precisa de la carga de la enfermedad.
Esta es la primera investigación sobre epidemiología, curso clínico y desenlaces del dengue grave en menores de 18 años, provenientes de áreas de alta transmisión del virus, que se realiza en una institución de salud pediátrica de Cali, Colombia.

Con respecto a la mediana de edad para el total de la población a estudio, que correspondió a 9,3 años, los datos difieren con otros estudios, con hallazgos que varían entre 5 a 10 años (13). En el estudio de Sujatha et al., se describe que el 72,7\% de los niños con dengue se encontraban por debajo de los nueve años de edad (14). Por otro lado, el estudio de Salgado et al., incluye pacientes con miocarditis por dengue, donde la mediana de edad fue de 72 meses (seis años) para este grupo de pacientes (15). Adicionalmente, la edad superior a seis años, como variable asociada a dengue severo, fue reportada en el estudio de Pothapregada $S$ et al (16).

La seroprevalencia del 77,2\%, fue similar a lo reportado por Rojas et al. (17), realizado en población pediátrica de Cundinamarca. Se requieren realizar extensos estudios serológicos de la seroprevalencia de anticuerpos contra el virus del dengue para proporcionar una estimación más precisa de la carga de la enfermedad (9). Los datos de vigilancia son extremadamente importantes para la identificación de las tendencias de la enfermedad y los datos disponibles indican una tendencia cambiante en la naturaleza de la enfermedad en Colombia, durante el período de revisión (18)(19).

La infección por cualquier serotipo DENV induce un amplio espectro de manifestaciones que van desde la ausencia de síntomas hasta la muerte (18). Aunque un gran porcentaje de los casos son asintomáticos, en los casos sintomáticos, la severidad del cuadro clínico podría depender de factores como el serotipo viral, la virulencia de la cepa, del estado nutricional, los factores genéticos del hospedero y la historia de infecciones previas con otros serotipos de DENV (1)(20)(21). 
Mayo - agosto de 2020 - Pág 99

La identificación temprana del dengue es un pilar para la prevención y la atención oportuna de sus complicaciones, que ocurren usualmente hacia el quinto día de enfermedad.
A diferencia de la población adulta, en la que el ingreso a unidades de cuidado intensivo está asociado directamente con la edad y las comorbilidades asociadas (22), en pediatría, dado el perfil clínico fluctuante del dengue es difícil predecir su evolución; no obstante, durante la vigilancia clínica y hemodinámica de cada paciente, es importante conocer cuáles manifestaciones clínicas suelen estar más asociadas a ingreso a unidad pediátrica de cuidados intensivos.

En el estudio realizado por Shrishu K et al., las indicaciones más frecuentes para ingreso a unidad pediátrica de cuidados intensivos, que hacen parte del espectro de dengue grave, fueron el choque persistente no corregido, seguido de dificultad respiratoria y síntomas neurológicos; así mismo, se describe que el síndrome compartimental abdominal, contribuye a la refractariedad del choque (23). Similar a lo evidenciado en este estudio, Dhochak et al. reportan que los síntomas gastrointestinales hacen parte de las manifestaciones mayormente asociadas a ingreso a unidad pediátrica de cuidados intensivos (24). Pothapregada $S$ et al., describen que los signos de alarma más asociados a dengue severo en el estudio realizado en Puducherry, India, fueron dolor abdominal, hepatomegalia e hipotensión (16).

En el estudio de Salgado et al. (21) se describen las causas de mortalidad por dengue hemorrágico en niños menores de 13 años en Neiva, Colombia. De 1448 niños con dengue hemorrágico, 23,3\% ingresaron a la UCIP y hubo una letalidad del 1,03\%. Los síntomas gastrointestinales y signos de fuga vascular estuvieron presentes en todos los casos, lo cual es consistente con lo hallado en el presente estudio, dado que los tres pacientes fallecidos presentaron dolor abdominal y signos de fuga vascular.

Se debe resaltar que en pacientes con infección secundaria, la probabilidad de que se presente dengue grave es mayor, por el fenómeno conocido como amplificación dependiente de anticuerpos (25). En el presente estudio dos de los tres pacientes fallecidos presentaron IgG positivo, así mismo, del grupo de casos, el 70,8\% presentó infección secundaria.

La identificación temprana del dengue es un pilar para la prevención y la atención oportuna de sus complicaciones, que ocurren usualmente hacia el quinto día de enfermedad (26)(27). Por lo anterior, el retraso en su diagnóstico puede tener implicaciones sobre el pronóstico, dado que el inapropiado manejo en los primeros días puede ser un factor determinante de la aparición de complicaciones potencialmente letales (28)(29)(30).

Dentro de las limitaciones del estudio, es importante aclarar que es un estudio unicéntrico, en un periodo de dos años y el escenario es de una institución clínica privada que brinda servicios en un $85 \%$ al régimen subsidiado en salud y $15 \%$ al régimen contributivo, los hallazgos deben ser tomados con prudencia al ser extrapolados a otras instituciones.

La naturaleza retrospectiva de esta investigación y el análisis secundario, podrían tener sesgos implícitos en el manejo de la información, dado que esta fue recolectada para la prestación de servicio de salud y no para fines investigativos.

\section{Conclusiones}

Las manifestaciones clínicas inespecíficas del dengue pediátrico lo convierten en un reto para el profesional de salud, dado que si no se realiza un correcto abordaje clínico puede culminar en desenlaces fatales. Sin embargo, identificando tempranamente los 
factores de riesgo descritos y un manejo ajustado a la severidad, con traslado oportuno a unidad pediátrica de cuidados intensivos, los desenlaces fatales pueden ser reducidos.

\section{Agradecimientos}

A todo el personal de la Fundación Clínica Infantil Club Noel por hacer posible la realización de este trabajo y a los estudiantes de Medicina y Pediatría de la Universidad Libre, seccional Cali, por su colaboración en la obtención de los datos las historias clínicas.

\section{Bibliografía}

1. Castellanos J, Bello J, Velandia-Romero M. Manifestaciones neurológicas durante la infección por el virus del dengue. Infectio. 2014;18(4).

2. Ly S, Fortas C, Duong V, Benmarhnia T, Sakuntabhai A, Paul R, et al. Asymptomatic Dengue Virus Infections, Cambodia, 2012-2013. 2019;25(7):2012-3.

3. Mustafa MS, Rasotgi V, Jain S, Gupta V. Discovery of fifth serotype of dengue virus (denv-5): A new public health dilemma in dengue control. Med J Armed Forces India. 2015;71(1).

4. Restrepo BN, Piedrahita LD, Agudelo IY, Parra-Henao G, Osorio JE. Frequency and clinical features of dengue infection in a schoolchildren cohort from Medellin, Colombia. J Trop Med. 2012;2012:120496.

5. Rojas J, Palacios R, Alzate A, Concha-eastman Al. La etnia afrocolombiana, factor protector paradójico contra el Dengue. Colomb Med. 2016;47(3):133-41.

6. Cuéllar-Jiménez ME, Velásquez-Escobar OL, González-Obando R, Morales-Reichmann CA. Detección de Aedes albopictus (Skuse) (Diptera: Culicidae) en la ciudad de Cali, Valle del Cauca, Colombia. Biomédica. 2014;27(2):273.

7. Minsalud, OMS/OPS. Protocolo para la Vigilancia en Salud Pública del Dengue. Anexo pediatrico guía clínica dengue. Convenio 637/09 [Internet]. Organización Panameriaca de la Salud, Organización Mundial de la Salud, Ministerior de Protección Social. 2010 [cited 2015 Sep 20]. Available from: http://www.ins.gov.co/ temas-de-interes/Dengue/02 D Anexo Pediatrico Dengue.pdf

8. Dalrymple NA, MacKow ER. Virus interactions with endothelial cell receptors: Implications for viral pathogenesis. Curr Opin Virol. 2014;7(1).

9. Villar LA, Rojas DP, Besada-Lombana S, Sarti E. Epidemiological Trends of Dengue Disease in Colombia (2000-2011): A Systematic Review. PLoS Negl Trop Dis [Internet]. 2015;9(3):e0003499. Available from: http://dx.plos.org/10.1371/journal. pntd.0003499

10. Rojas JH. Boletín epidemiológico No. 10 semana epidemiológica No. 39. Secretaría de Salud Municipal de Cali. [Internet]. 2016. Available from: www.cali.gov.co/salud/publicaciones/descargar.php?id=45039

11. Organización Panamericana de la Salud. Dengue: Guías de atención para enfermos en la región de las Américas [Internet]. 2010. 56 p. Available from: http://www. paho.org/hq/index.php?option=com docman\&task=doc view\&gid=11239\&/temid 
12. Páez A, Herrera VM, Gómez AM, Mantilla JC, Miranda MC, Rojas DP, et al. Clinical Indicators of Fatal Dengue in Two Endemic Areas of Colombia: A Hospital-Based Case-Control Study. Am J Trop Med Hyg. 2019;100(2):411-9.

13. Bhave S, Rajput C. Clinical profile and outcome of dengue fever and dengue haemorrhagic fever in paediatric age group with special reference to WHO guidelines (2012) on fluid management of dengue fever. Int J Adv Res. 2015;3:196-201.

14. Ramabhatta S, Palaniappan S, Hanumantharayappa N, Begum SV. The Clinical and Serological Profile of Pediatric Dengue. Indian J Pediatr. 2017:84(12):897-901.

15. Salgado DM, Panqueba CA, Castro D, R. VM, Rodríguez JA. Miocarditis en Niños con Fiebre por Dengue Hemorrágico en un Hospital Universitario de Colombia. Rev Salud Pública. 2008;(0124-0064).

16. Pothapregada S, Sivapurapu V, Kamalakannan B, Thulasingham M. Role of early warning signs in children with severe dengue infection. Int J Contemp Pediatr. 2018;5(4):1423.

17. Rojas J, Alvarez M, Castellanos J, Velandia M, Coronel C. Caracterización de Dengue en menores de 14 años en el departamento de Cundinamarca en Colombia. [Internet]. Universidad El Bosque, Bogotá-Colombia; 2016. Available from: http://catalogo.unbosque.edu.co/uhtbin/cgisirsi.exe/?ps=D7BpjSESIO/BIBLIOBOSQ/66220056/9

18. Rosso F, Vanegas S, Rodríguez S, Pacheco R. Prevalencia y curso clínico de la infección por dengue en adultos mayores con cuadro febril agudo en un hospital de alta complejidad en Cali , Colombia. 2016;36.

19. Instituto Nacional de Salud de Colombia. Contenido Notificación [Internet]. Semana epidemiológica número 46 de 2015 (15 nov. al 21 nov.). 2015. Available from: http://www.ins.gov.co/boletin-epidemiologico/Boletn Epidemiolgico/2015 Boletin epidemiologico semana $\underline{46 . p d f}$

20. Paranavitane SA, Gomes L, Kamaladasa A, Adikari TN, Wickramasinghe N, Jeewandara C, et al. Dengue NS1 antigen as a marker of severe clinical disease. BMC Infect Dis [Internet]. 2014;14(1):1-7. Available from: http://www.biomedcentral.com/1471-2334/14/570

21. Salgado DM, Rodríguez J a., Garzón M, Cifuentes G, Ibarra M, Vega MR, et al. Caracterización Clínica y Epidemiológica de Dengue Hemorrágico en Neiva, Colombia, 2004. Rev Salud Pública [Internet]. 2007;9(1):53-63. Available from: http://www. scielosp.org/scielo.php?script=sci_arttext\&pid=S0124-00642007000100006\&l$\underline{\text { ng=es\&nrm=iso\&tlng }=e s}$

22. Hsieh CC, Cia CT, Lee JC, Sung JM, Lee NY, Chen PL, et al. A Cohort Study of Adult Patients with Severe Dengue in Taiwanese Intensive Care Units: The Elderly and APTT Prolongation Matter for Prognosis. PLoS Negl Trop Dis. 2017;11(1):1-14.

23. Kamath SR, Ranjit S. Clinical features, complications and atypical manifestations of children with severe forms of dengue hemorrhagic fever in South India. Indian J Pediatr. 2006;73(10):889-95. 
24. Nitin D, Rakesh L. Dengue in children: Issues in critical care settings. J Pediatr Crit Care. 2017;4(3):44-53.

25. Martínez J, Hernández JC, Urcuqui Inchima S. Papel de las células dendríticas en la infección por el virus dengue: blancos de replicación y respuesta inmune. Rev Chil infectología. 2017;34(3):249-56.

26. Salgado DM, Castro D. Mortalidad por dengue hemorrágico en niños en Colombia: más allá del choque. Asoc Colomb Infectología. 2008;12(1):21-7.

27. Minsalud, OPS, INS. Guía Para La Atención Clínica Integral Del Paciente Con Dengue. Bogotá: Minsalud, Organización Panamericana de la Salud, Instituto Nacional de Salud; 2010.

28. Tuan NM, Nhan HT, Chau NVV, Hung NT, Tuan HM, Tram TVan, et al. Sensitivity and Specificity of a Novel Classifier for the Early Diagnosis of Dengue. PLoS Negl Trop Dis [Internet]. 2015;9(4):e0003638. Available from: http://dx.plos.org/10.1371/ journal.pntd.0003638

29. Organización Mundial de la Salud, OPS. Actualización Epidemiológica Dengue. In: OMS, editor. Organización panameriaca de la Salud. Washington: OPS/OMS; 2019.

30. Minsalud, INS. Informe De Evento Dengue, Colombia, 2017. Organ panameriaca la Salud [Internet]. 2017;14(1):1-22. Available from: https://www.ins.gov.co/buscador-eventos/Informesdeevento/DENGUE 2017.pdf 